\title{
Home appliances controller using wireless controller area network (WCAN) system
}

\begin{abstract}
This paper presents a wireless remote controller in which providing a wireless sensing solution for home users to operate essential household appliances, ranging from simple lightings to sophisticated electronic devices. A wireless protocol based on wireless token ring protocol (WTRP) has been modified, yielding wireless controller area network (WCAN) data centric communications, which is then proposed to be applied in managing concurrency control of home appliances. WCAN is an adaptation of its wired cousin, controller area network (CAN) protocol which has not being properly defined. The wireless controller communicates with the standalone server which in turn communicates with the rest of sensing nodes through WCAN system. Each node will be receiving command from the standalone server and based on the message identifier, either executing the command or discarding it. WCAN has been chosen to become the backbone network of the system as it offers an alternative solution when industrial mobile stations under certain constraints should continue to use CAN protocol as frame exchange protocol.
\end{abstract}

Keyword: CAN; WTRP; Home appliances; Standalone server 